\title{
Impacts of River Groove Propagation on Irrigation Infrastructural Failures
}

\author{
Kuntjoro $^{1}$, Choirul Anwar ${ }^{1}$, and Didik Harijanto ${ }^{1}$
}

\begin{abstract}
Water supply of irrigation intakes are generally from the river. The system considers the lowest water level of the river on its mechanism. River groove propagation is a development of erosion at some parts and at the same time a development of sedimentation at the other parts of the river bed. This phenomenon is identified as an indication that cause the flood which might endangers the surrounding infrastructures. Analysis by KUN-QARSHOV method on Brantas river groove propagation in Mojokerto areas is acquired. Most progressive vertical meander shifting is at cross section KB63. The shift isas deep as 0.38 metres per year. The second progressive vertical meander shifting is at cross section KB64. The shift is as deep as 0.27 metres per year. The most progressive horizontal meander shifting is at cross section KB64. The horizontal shift moves toward the right cliff at 0.17 metres per year. The second progressive horizontal meander shifting occurs at cross section KB63. The horizontal shift moves toward the right cliff at 0.14 metres per year. The groove propagation could be more dangerous if there is a combination between vertical and horizontal shifts at the same time that might cause severe cliff slides. The failure of the irrigation sytem at Brantas River in Mojokerto areas was due to the water level of the river below the irrigation intake level. Among others are the intakes at Keboan, Ngareskidul, and Gedeg irrigation areas. Another is due to the erosion of river bed at the syphon of Watudakon irrigation area.
\end{abstract}

Keywords -irrigation system, meander shifting, river groove propagation.

\section{INTRODUCTION}

$\mathrm{W}$ ater supply of irrigation intakes are generally from the river. The system considers the lowest water level of the river on its mechanism. River groove propagation is a development of erosion at some parts and at the same time a development of sedimentation at the other parts of the river bed. This phenomenon is identified as an indication that cause the flood which might endangers the surrounding infrastructures. Discharge quantity, bank resistance, flat topography, and characteristics at certain locations of the Brantas river flow have led to the Brantas river meanders formation. At the river meanders, the threat against the infrastructures becomes more real than at the other straight parts of the river. It can be seen that the groove propagation in meandering river is faster than in a relatively straight river. In Menturus-Mojokerto segment, the river groove propagation has already threatened some of the irrigation intakes.

Several previous proposed models to forecast the river meander movement or river propagation can be found in literatures [1-2,8-10,14-15, and 17]. This study is carried out to obtain the relationships of the meander movement velocity changes, the influencing parameters which can be obtained through analytical models, the regression techniques in which the graphical representations produces an equation (the graph should be suitable for estimating the meander movement rate).

\section{METHOD}

The river groove propagation at Menturus-Mojokerto's Brantas river segment is more likely due to the discharge fluctuation change [13-14].

${ }^{1}$ Kuntjoro, Choirul Anwar, and Didik Harijanto are with Department of Civil Engineering, Faculty of Civil Engineering and Planning, Institut Teknologi Sepuluh Nopember, Surabaya, 60111, Indonesia. E-mail: kuntjoro_rivers@yahoo.co.id; choirulanwar.its@gmail.com; didi_hari@yahoo.com.
In the paper, the river groove propagation at MenturusMojokerto segment can be predicted by using KUNQARSHOV method. The result is compared with the measuring data. The water level prediction is also compared with the water level data obtained from measurement in this segment.

Figure 1 shows the geometrical movement of the river which is analyzed at each movement point of a cross section. Each point is represented by Node $(x i, y i)$, where $i=1,2, \ldots, n$ at every second the discharge passing through the point. Each point will shift to a new Node $\left(x i^{\prime}, y i^{\prime}\right)$. The original Node $(x i, y i)$ will become a new one (xi', yi') and the increment will be $(\Delta x i, \Delta y i)$. Where $\Delta x i$ is the distance of the horizontal movement from point $x i$ to $x i$ ' and $\Delta y i$ is the distance of vertical movement from point $y i$ to $y i$ '.

A. Inception of Channel Meandering [19]

The magnitude of the basal erosion can be analyzed using the rate of precipice erosion speed per unit of river length per unit of time. The mass volume enters into the river from the basal erosion can be calculated by the following equation:

$q_{b r}^{b}=\frac{\bar{\xi}(1-p) h_{b}}{\sin \bar{\beta}}$

where $q_{b r}^{b}$ is the net sediment volume towards the main river from precipices erosion, and $h_{b}$ is the depth of the flow near the cliffs and $p$ is the porosity of river precipicematerial.

B. Numerical simulation of gravel river widening

The erosion occurred at the bottom of the river and at the river bank below the water surface will decrease the river bank. This will then tear down the river bank above the water surface and might lead to the increase of the angle of material response to exceed the limit. The amount of material from the collapse of the cliff is calculated using the following equation:

$$
q_{b r}^{f}=\bar{\xi} \Delta h_{b a n k}(1-p)
$$

where $q_{b r}^{f}$ is the eroded sediment materials per unit of channel width from the collapse of the cliff, and $\Delta h_{b a n k}$ is the height of the cliff above the water surface. 


\section{Meander Parameter}

This research was conducted at Brantas river meanders in Mojokerto area using the analyzed river geometry map. Meander curves at each bend of the river were marked with Notations R1 to R6. This map analysis was conducted to obtain the meander parameters. The detailof the map analysis is shown in Figure 2. The measured segment of the observed river length was as long as $6,380 \mathrm{~m}$ at the meander axis of $3,290 \mathrm{~m}$.

D. River Geometrical Change Equation

QARSHOV Method)

River geometrical change equation was constructed by considering the sedimentation function, $E=f\left(Q, A, h_{b}\right.$, $O, S, r_{c}, \phi, \theta, \lambda, a$ and $\left.t\right)$ and scouring function, $G=f(Q$, $A, h_{b}, O, D_{50}, r_{c}, \phi, \theta, \lambda, a$ and $\left.t\right)$.

where: $Q$ : discharge rate

$$
\begin{aligned}
& A: \text { wet area } \\
& h_{b}: \text { height of water level from the bottom } \\
& O: \text { wet circumference } \\
& S: \text { sediment concentration } \\
& r_{c}: \text { radius of meander curvature } \\
& \phi: \text { bend angle } \\
& \theta: \text { relative angle }(0<\theta<\phi) \text { within each bend } \\
& \lambda: \text { meander length } \\
& D_{50}: \text { mean sediment diameter } \\
& a: \text { meander amplitude } \\
& t: \text { time step }
\end{aligned}
$$

Sedimentation rate per unit of time $(E)$ can be determined by the following equation:

$E=C_{E} \frac{Q A S}{V O} \frac{h_{b}}{r_{c}^{3}} \frac{\theta}{\phi} \frac{a}{\lambda} \frac{1}{t}$

where $C_{E}$ : sedimentation coefficient depends on $\theta / \phi$ and $\alpha / \lambda$ values.

Scouring rate per unit of time $(G)$ can be determined by an equation as follows:

$$
G=C_{G} \frac{Q A D_{50}}{V O} \frac{h_{b}}{r_{c}^{4}} \frac{\theta}{\phi} \frac{a}{\lambda} \frac{1}{t}
$$

where $C_{G}$ : scouring coefficient depend on $\theta / \phi$ and $\alpha / \lambda$ values.

The movement directions of each point at the river bottom include the horizontal and vertical movements of the points. $\Delta \mathrm{h}$ and $\Delta \mathrm{v}$ are different for $E$ and $G$. They can be written by $\Delta h=h_{0}+(E-G)$ and $\Delta v=v_{0}+(E-G)$. If $\Delta h$ and $\Delta v$ are positive then the sedimentation occurs, and if negative, the erosion takes place.

\section{RESULT AND DISCUSSION}

The prediction of river groove propagation and the measurement data in 1997 atbenchmark cross section No. KB64 is listed in Table 1 and illustrated in Figure 3. The predictions in 2001, 2006, 2008, and 2011 are also given in Figures 4 to 7 , respectively. The condition of irrigation intake isshown in Figure 8. The corresponding data for cross section No. KB63 is also provided in Table 2.

Erosion at benchmark No. KB64 is more dominant at the bottom of the river. The erosion at the left and right sides of the river seemed to be balanced. The cross section at benchmark No. KB63 shows thatthe erosion of right side of the river is larger than the left. The meanders are expected to shift towards the left bank at this cross section.
The most vertical progresive meander shifting is atbenchmark cross section No. KB63 during the 1992 to 2011 interval. The shift is as deep as 7,27 m.

The second progressive vertical meander shifting is atbenchmark cross section No. KB64 $\left(\theta / \phi=60^{\circ} / 165^{\circ}\right.$, and $r_{c}=357 \mathrm{~m}$ ). During the interval of 1992 to 2011, the shift occurred as deep as $5.05 \mathrm{~m}$. The cross section is the beginning part of the meanders, hence the greatest erosion occurred at this section.

The most progressive horizontal meander shifting occurredatbenchmark cross section No. KB64. The horizontal shift moved towards the right cliff as much as 3.15 meters.

The second progressive horizontal meander shifting was occurred at cross section benchmark No. KB63. The horizontal shift moved towards the right bank as far as 2.67 metres.

\section{CONCLUSION}

The river groove propagation lowered water surface of the river. The failure occurred at some irrigation infrastructures due to the lower water surface of the river from the intake threshold. Among others are the intakes of Keboan, Ngareskidul, Gedeg irrigation areas.

The river bed degradation occurred and might be further threatening the syphon infrastructure of Watudakon irrigation area.

\section{ACKNOWLEDGEMENT}

The authors would like to acknowledge their gratitude to the officials and staffs of the JasaTirta I in Malang and the "BalaiBesar Wilayah Sungai Brantas" in Surabaya for the facilities, generosity, services, and supporting data, which makes this research possible.

\section{REFERENCES}

[1] Keady D. M. and Priest M. S., "and , "The Downstream Migration Rate of River Meandering Patterns," in Mississippi Water Resources Conference, Meeting 12th Mississippi Water Resources Conference, Jackson, MS, 1977, pp. 29-34.

[2] A. J. Odgaard, "Stream Bank Erosion along Two Rivers in Iowa," Water Resources Research 1987

[3] Pizzuto J. E. , "Numerical Simulation of Gravel River Widening," Water Resources, Research 361990

[4] Chien N. and Wan Z. H. , Dynamics of Sediment Transport. Beijing: Academic Press of China, 1991.

[5] Chih Ted Yang, Sediment Transport, Theory and Practice,' McGraw-Hill Companies, 1996.

[6] M. Morisawa, Rivers, 1st ed. New York: Longman, 1985.

[7] P. Y. Julien, River Mechanics, 1st ed. Cambridge, United Kingdom: Cambridge University Press, 2002.

[8] Jansen P. Ph., L. van Bendegom, J van de Berg, M de Vries, and A. Zanen, Principles of Rover Engineering, 1st ed.: Pitman Publishing Limited, 1979

[9] Biedenharn D. S. et al., "Relationship between Channel Migration and Radius of Curvature on the Red River," in Int. Symp. on Sediment Transport Modeling, New Orleans, LO, 1989, pp. 536-541.

[10] Briaud J. L. et al., "Erosion Function Apparatus for Scour Rate Predictions ," Journal of Geotechnical and Geoenvironmental Engineering, vol. 127, no. 2, pp. 105-113, 2001c.

[11] Brice J. C., "Lateral Migration of the Middle Sacramento River , U.S. Geological Survey, California, Water-Resources Investigations 1977

[12] Chang H. H. , "Analysis of River Meanders," Journal of Hydraulic Engineering, vol. 110, no. 1, pp. 37-50, 1984.

[13] Kuntjoro , M. Bisri, A. Masrevaniah, and A. Suharyanto, "Empirical Model of River Meandering Geometry Changes due to Discharge Fluntuation," Journal of Basic and Applied Scientific Research, vol. 2, no. 2, pp. 1027-1033, 2012 
[14] Kuntjoro , M. Bisri, A. Masrevaniah, and A. Suharyanto, "Modeling of discharge fluctuation influence on river meandering geometry change ," International Journal of Academic Research Part A. [Online], vol. 4, no. 6, pp. 189-196, November 2012.

[15] J. M. Hooke, "Magnitude and Distribution of Rates of River Bank Erosion," Earth Surface Processes, vol. 5, no. 2, pp. 143 157, 1980.

[16] Hudson P. F. and Kesel R. H., "Channel Migration and Meander-bend Curvature in the Lower Mississippi River Prior to Major Human Modification," Geology, vol. 28, no. 6, pp. 531534, 2000.

[17] S. Ikeda, G. Parker, and K. Sawi, "Bend Theory of River Meanders. I: Linear Development," Journal of Fluid Mechanics, vol. 112, pp. 363-377, 1981.

[18] Nanson G. C. and Hickin E. J., "Channel Migration and Incision on the Beatton River," Journal Hydraulic Engineering, vol. 109, no. 3, pp. 327-337, 1983 .

[19] J. G. Duan, Simulation of Stream Bank Erosion Processes with a Two-Dimensional Numerical Model. In Landscape Erosion and Evolution Modeling, Harmon RS, Doe WW III ed. New York: Kluwer Academic/Plenum Publishers, 2001.

[20] J. G. Duan and Y. Julien Pierre. (2005) Numerical Simulation of The Inception of Channel Meandering,Earth Surf. Process. Landforms 30,[Online] Wiley Inter Science. [Online]. www.interscience.wiley.com

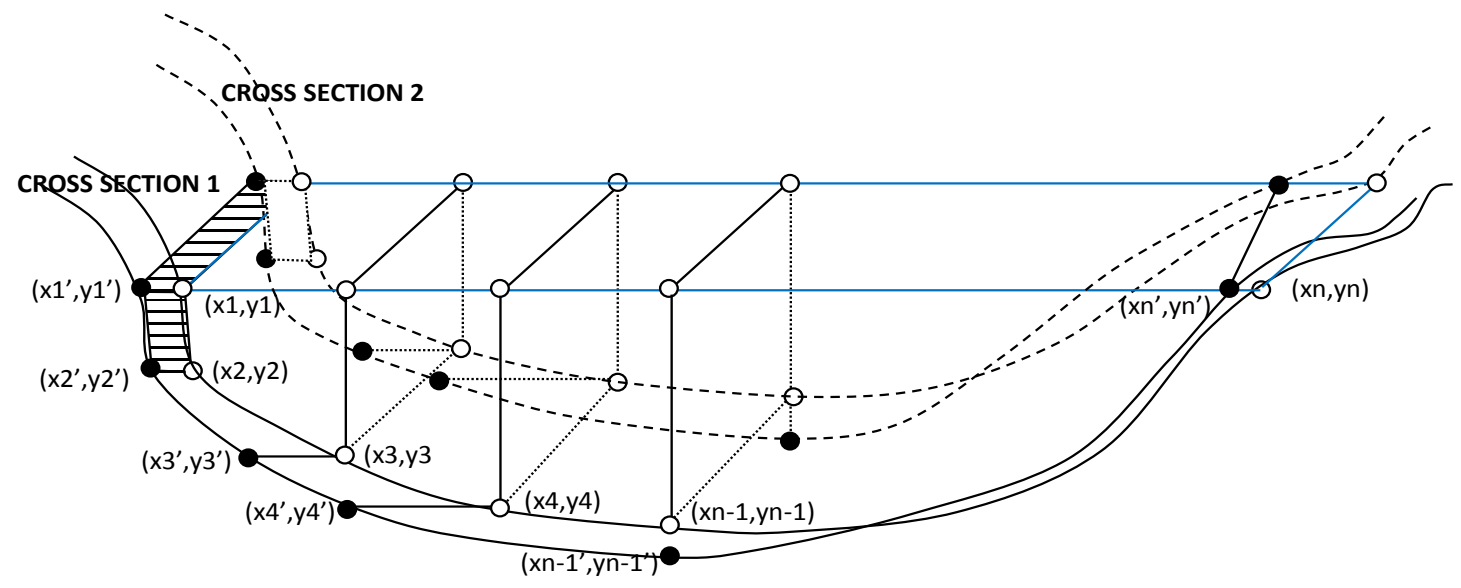

Figure 1.Nodes of river geometry movement [13-14]

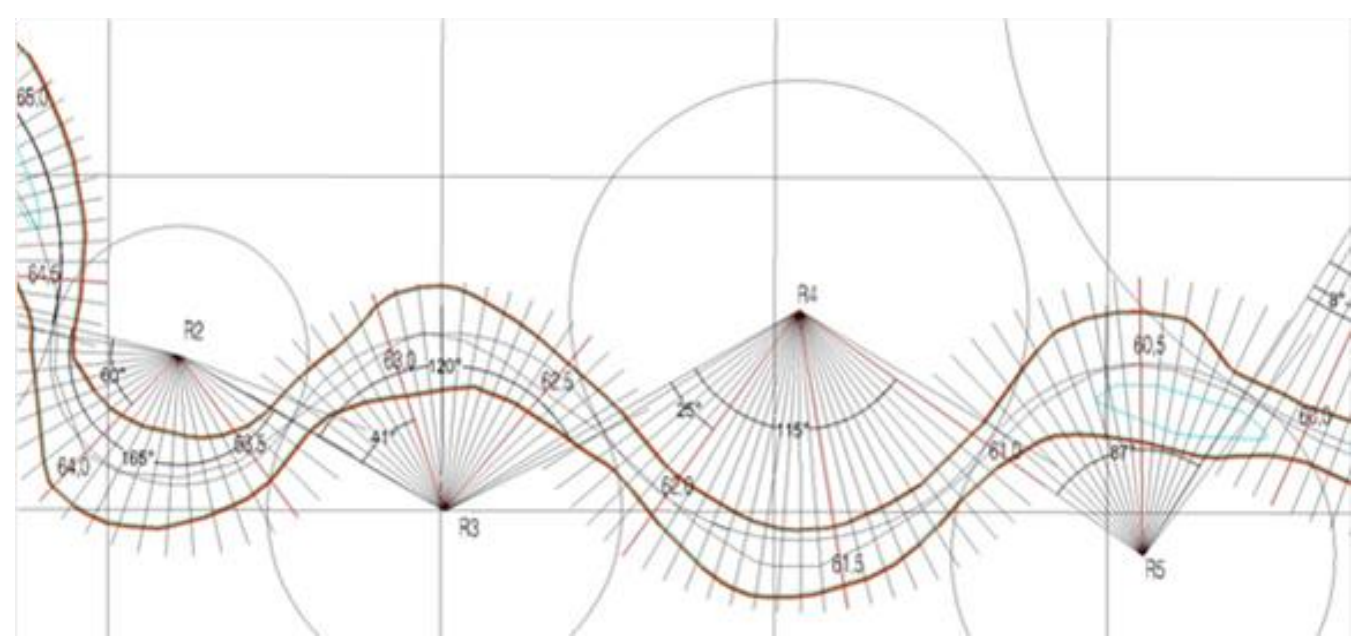

Figure 2. Meander parameters at Brantas river [13-14] 


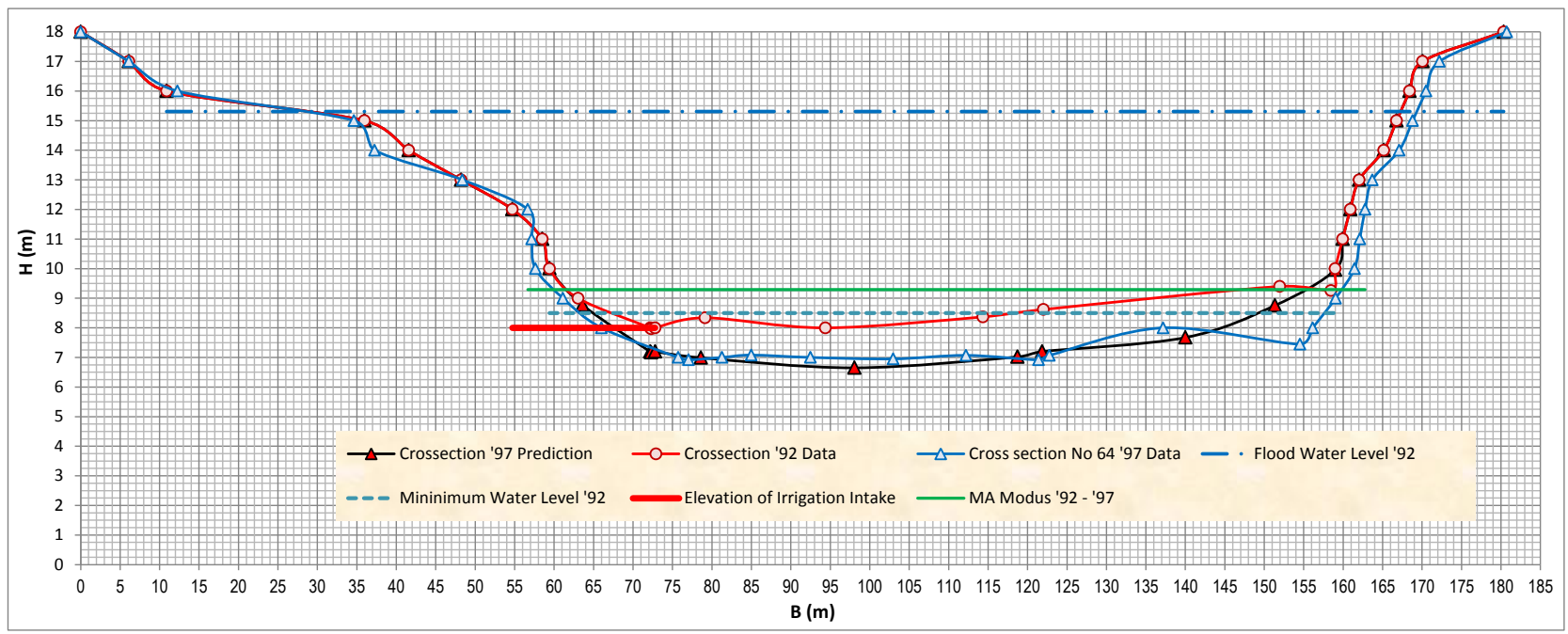

Figure 3.Comparison of minimum water level with intake elevation at Benchmark No. 64 in 1992

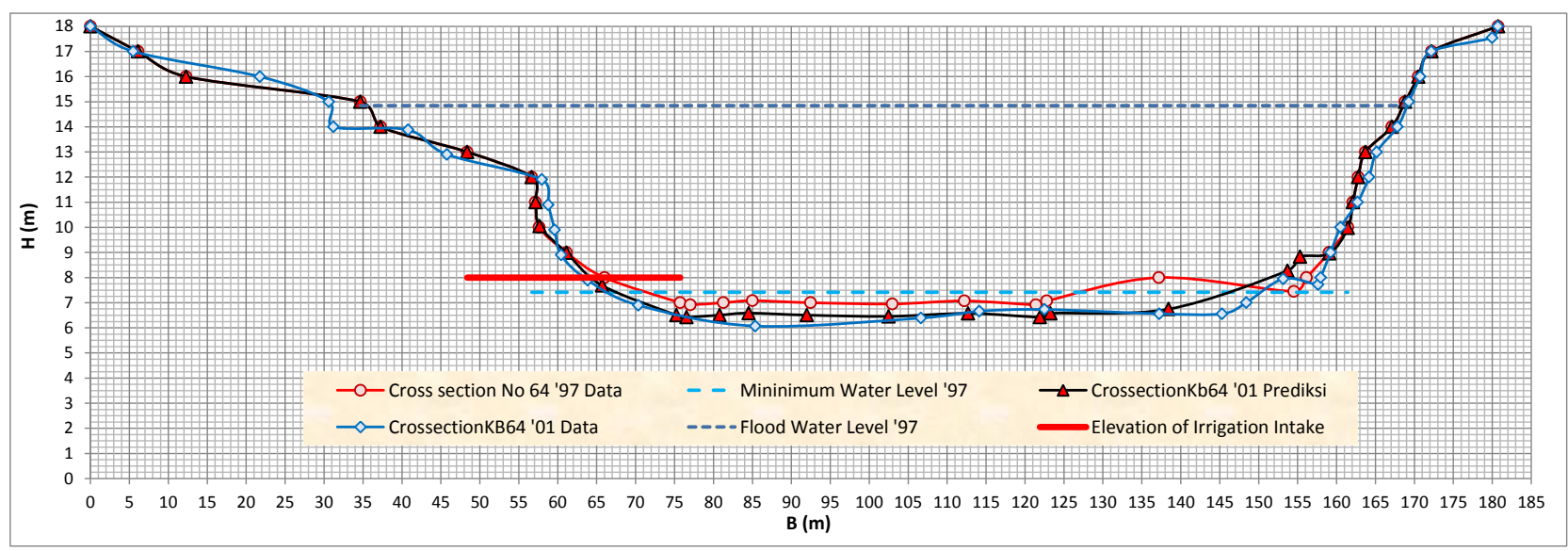

Figure 4.Comparison of minimum water level with intake elevation at Benchmark No. 64 in 1997

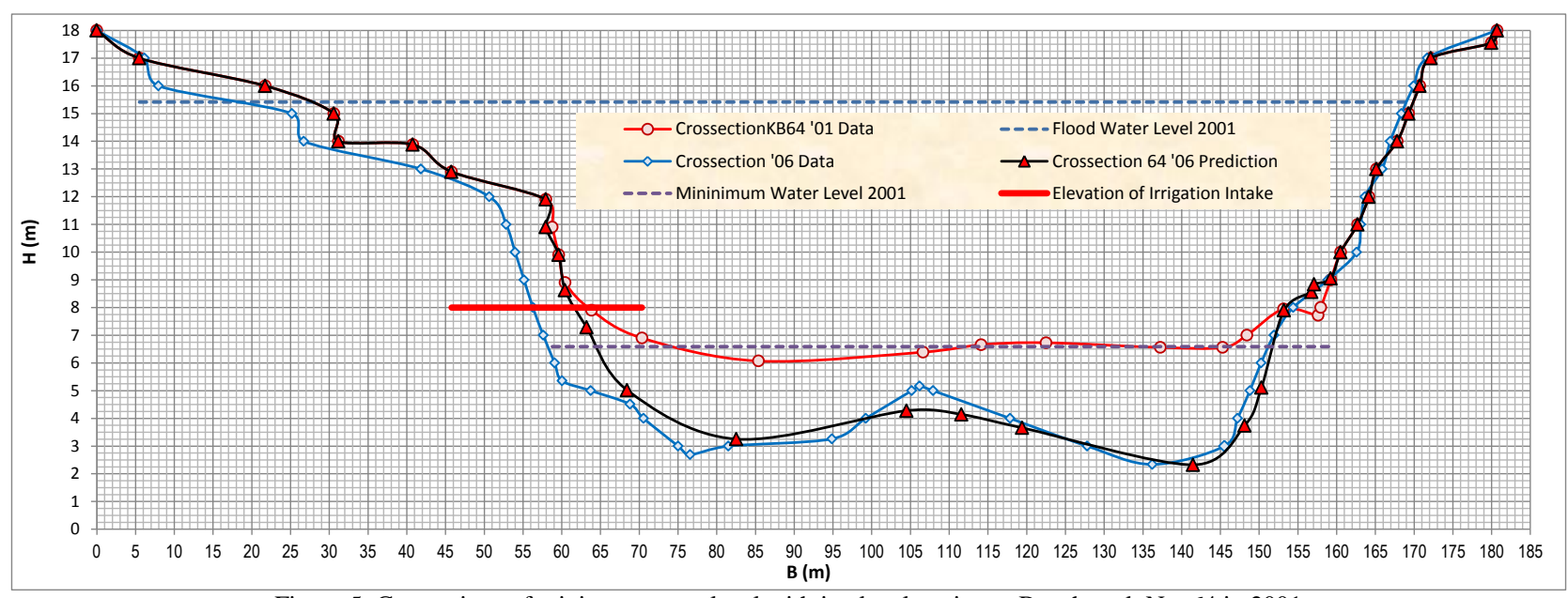

Figure 5. Comparison of minimum water level with intake elevation at Benchmark No. 64 in 2001 


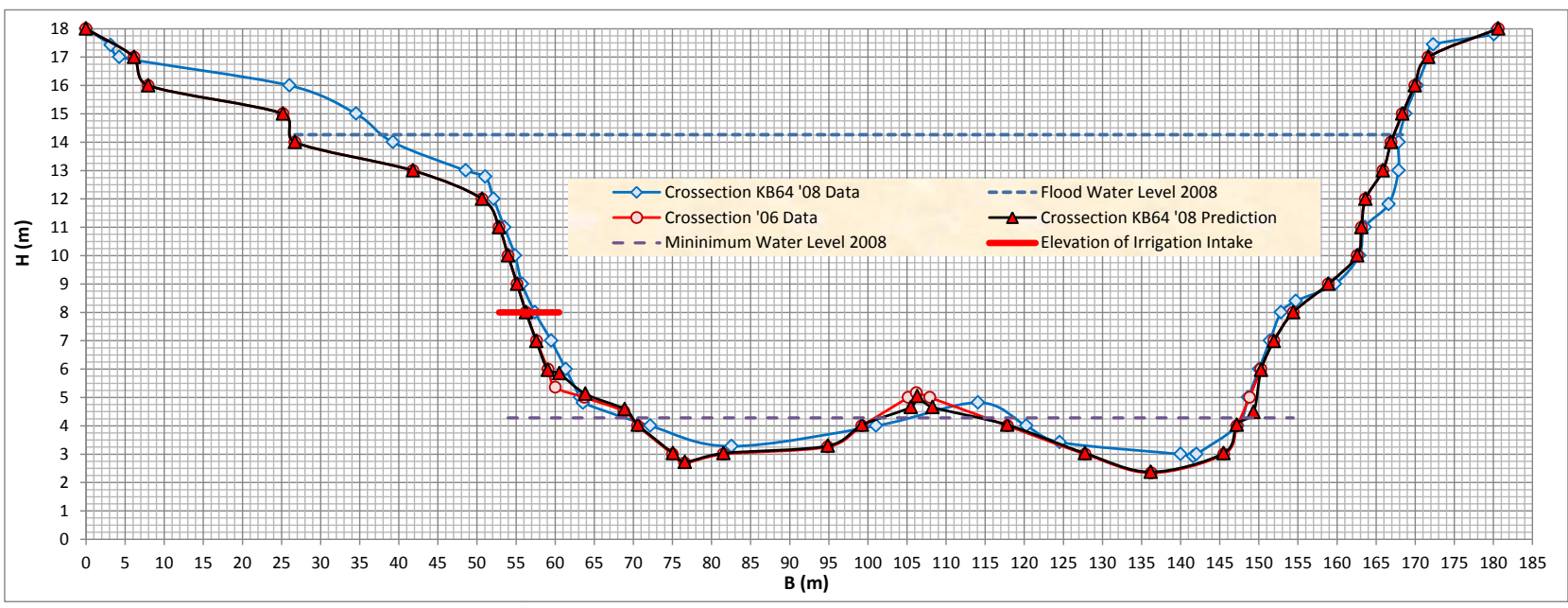

Figure 6. Comparison of minimum water level with intake elevation at Benchmark No. 64 in 2008

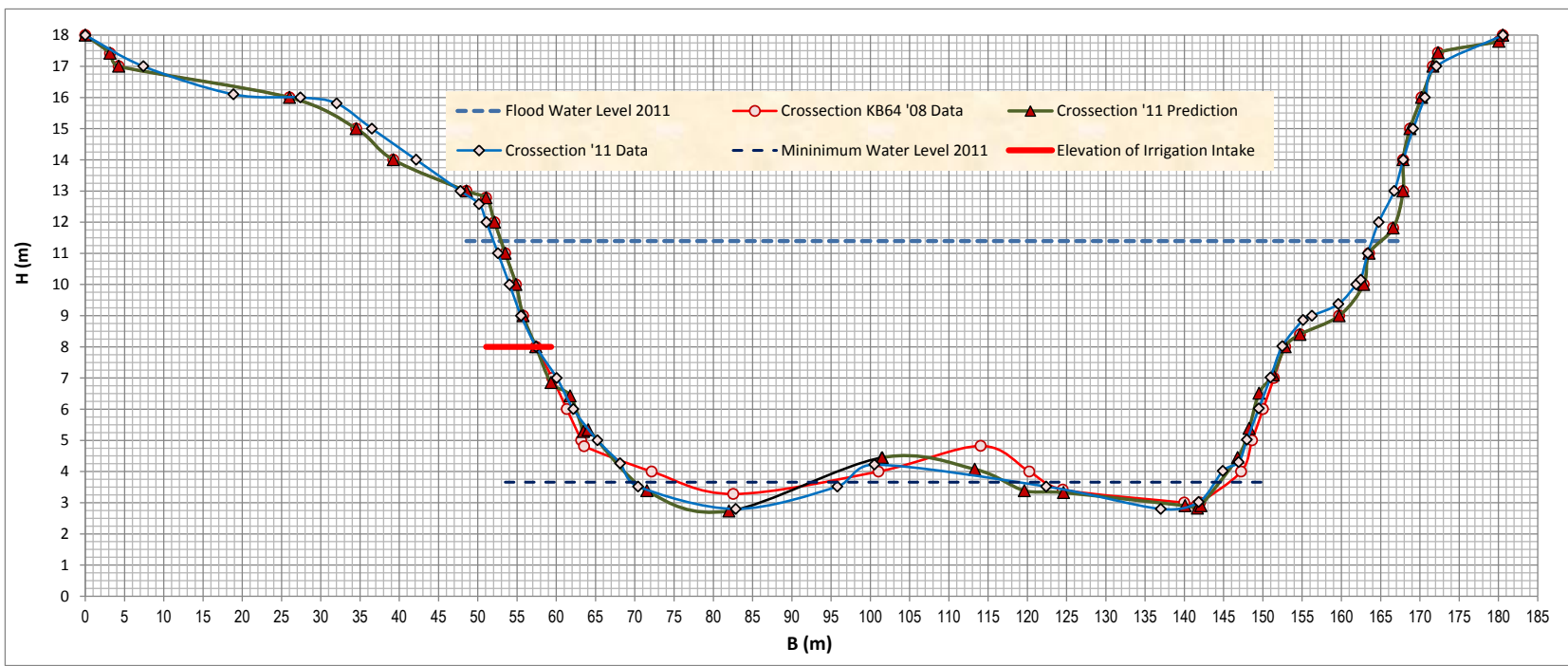

Figure 7. Comparison of minimum water level with intake elevation at Benchmark No. 64 in 2011

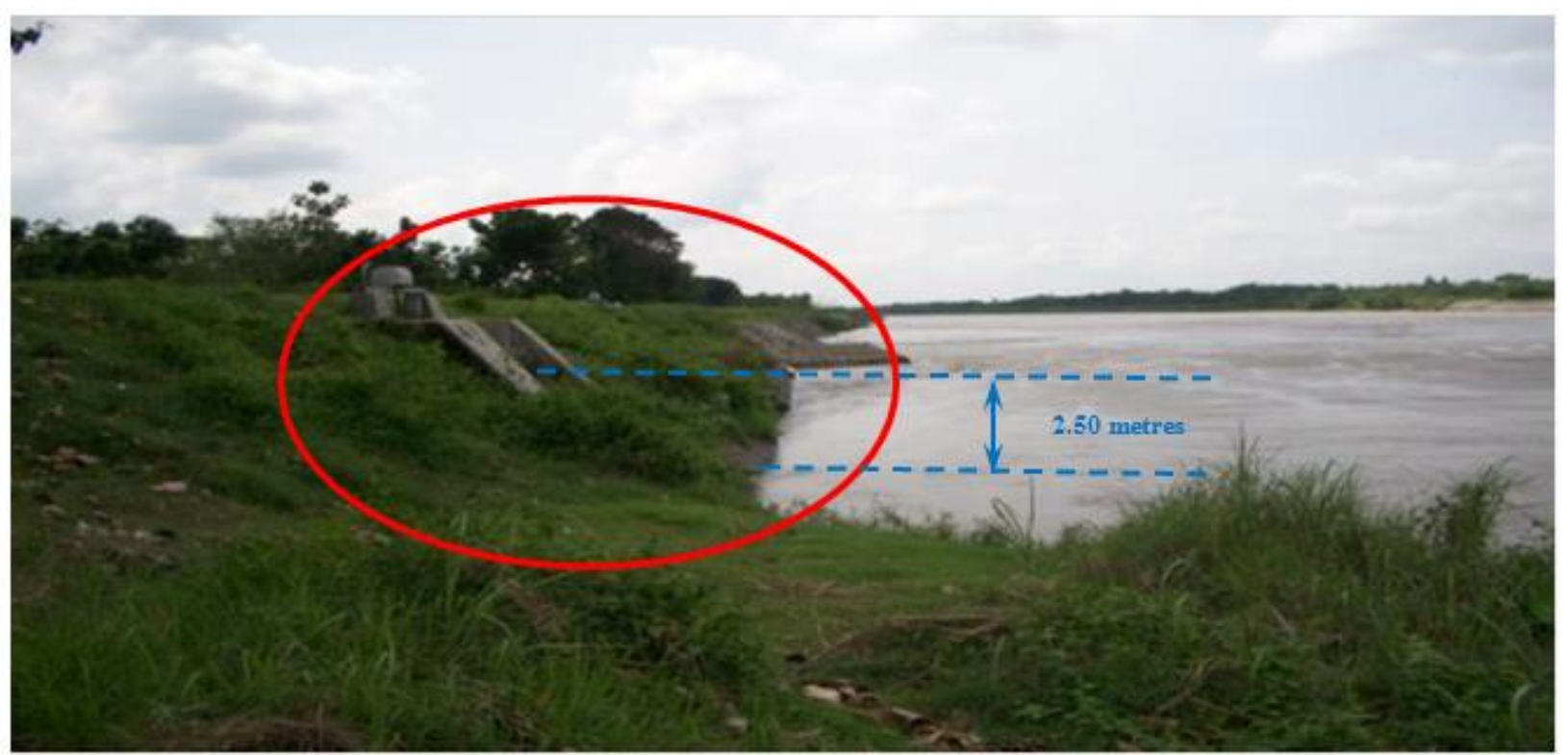

Figure 8. Water level below irrigation intake at downstream of Menturus rubber dam in June 2011 
TABLE 1.

COORdinates OF River Bed at CRoss Section No. KB64, DownStReAm OF MENTURUS RubBer DaM

\begin{tabular}{|c|c|c|c|c|c|c|c|c|c|c|c|c|c|}
\hline \multicolumn{2}{|c|}{$\begin{array}{c}\text { Measurement } \\
\text { '92 } \\
\end{array}$} & \multicolumn{2}{|c|}{ Prediction '97 } & \multicolumn{2}{|c|}{ Prediction '01 } & \multicolumn{2}{|c|}{ Prediction'06 } & \multicolumn{2}{|c|}{ Prediction '08 } & \multicolumn{2}{|c|}{ Prediction '11 } & \multicolumn{2}{|c|}{$\begin{array}{c}\text { Measurement } \\
\text { '11 } \\
\end{array}$} \\
\hline 0.00 & 18.00 & 0.00 & 18.00 & 0.00 & 18.00 & 0.00 & 18.00 & 0.00 & 18.00 & 0.00 & 17.79 & 0.00 & 17.79 \\
\hline 1.84 & 17.00 & 1.84 & 17.00 & 2.02 & 17.00 & 2.15 & 17.00 & 1.42 & 17.00 & 2.08 & 17.00 & 3.87 & 17.00 \\
\hline 4.51 & 16.00 & 4.51 & 16.00 & 4.54 & 16.00 & 4.79 & 16.00 & 3.78 & 16.00 & 5.85 & 16.00 & 5.95 & 16.00 \\
\hline 14.14 & 15.00 & 14.14 & 15.00 & 14.93 & 15.00 & 15.98 & 15.00 & 18.21 & 15.00 & 17.30 & 15.00 & 19.02 & 15.00 \\
\hline 22.60 & 14.00 & 22.60 & 14.00 & 21.94 & 14.00 & 20.24 & 14.00 & 19.25 & 14.00 & 18.41 & 14.00 & 20.55 & 14.00 \\
\hline 46.96 & 13.00 & 46.96 & 13.00 & 47.39 & 13.00 & 21.81 & 13.00 & 19.61 & 13.00 & 20.63 & 13.00 & 22.08 & 13.00 \\
\hline 56.71 & 12.00 & 56.71 & 12.00 & 57.87 & 12.00 & 25.88 & 12.00 & 19.97 & 12.00 & 24.20 & 12.00 & 23.61 & 12.00 \\
\hline 60.15 & 11.00 & 60.15 & 11.00 & 59.35 & 11.00 & 36.31 & 11.00 & 24.30 & 11.00 & 33.68 & 11.00 & 42.60 & 11.00 \\
\hline 61.64 & 10.00 & 61.64 & 10.00 & 60.82 & 9.99 & 56.74 & 10.00 & 65.50 & 10.00 & 65.57 & 10.00 & 64.50 & 10.00 \\
\hline 63.13 & 9.00 & 63.13 & 9.00 & 69.55 & 8.98 & 61.74 & 8.94 & 68.84 & 9.00 & 67.87 & 9.00 & 67.33 & 9.00 \\
\hline 74.71 & 8.00 & 75.49 & 8.78 & 134.50 & 7.44 & 66.71 & 7.93 & 71.13 & 8.00 & 70.10 & 8.00 & 70.89 & 8.00 \\
\hline 128.16 & 7.27 & 128.84 & 7.95 & 170.32 & 6.10 & 99.72 & 1.75 & 73.02 & 7.00 & 72.38 & 7.00 & 73.08 & 7.00 \\
\hline 184.79 & 8.00 & 184.61 & 8.18 & 190.82 & 7.44 & 127.15 & 0.52 & 74.48 & 6.00 & 75.58 & 6.00 & 75.27 & 6.00 \\
\hline 193.63 & 9.00 & 193.63 & 9.00 & 193.95 & 8.98 & 173.74 & 2.34 & 77.03 & 5.00 & 78.76 & 4.98 & 78.48 & 5.00 \\
\hline 195.73 & 10.00 & 195.73 & 10.00 & 196.04 & 9.99 & 197.30 & 10.00 & 80.38 & 4.00 & 83.17 & 3.56 & 81.94 & 4.00 \\
\hline 197.83 & 11.00 & 197.83 & 11.00 & 198.13 & 11.00 & 197.31 & 11.00 & 83.64 & 3.66 & 111.07 & 1.99 & 88.49 & 3.00 \\
\hline 199.95 & 12.00 & 199.95 & 12.00 & 200.23 & 12.00 & 200.41 & 12.00 & 89.27 & 3.69 & 127.96 & 0.98 & 108.27 & 2.00 \\
\hline 202.25 & 13.00 & 202.25 & 13.00 & 202.33 & 13.00 & 202.56 & 13.00 & 117.45 & 2.56 & 139.09 & -0.22 & 125.68 & 1.00 \\
\hline 204.55 & 14.00 & 204.55 & 14.00 & 204.43 & 14.00 & 205.52 & 14.00 & 149.54 & 0.73 & 167.94 & 0.57 & 142.94 & 0.00 \\
\hline 206.85 & 15.00 & 206.85 & 15.00 & 206.53 & 15.00 & 207.50 & 15.00 & 170.84 & 0.51 & 170.94 & 1.28 & 170.72 & 1.00 \\
\hline 209.15 & 16.00 & 209.15 & 16.00 & 208.63 & 16.00 & 209.48 & 16.00 & 173.85 & 2.02 & 174.06 & 2.19 & 173.95 & 2.00 \\
\hline 215.87 & 17.00 & 215.87 & 17.00 & 213.45 & 17.00 & 216.46 & 17.00 & 177.39 & 3.00 & 177.08 & 3.20 & 177.18 & 3.00 \\
\hline \multirow[t]{16}{*}{217.32} & 18.00 & 217.32 & 18.00 & 216.54 & 18.00 & 219.44 & 18.00 & 181.26 & 4.00 & 180.28 & 4.03 & 180.41 & 4.00 \\
\hline & & & & & & 213.78 & & 186.78 & 5.00 & 183.30 & 4.98 & 183.22 & 5.00 \\
\hline & & & & & & 216.42 & & 190.70 & 6.00 & 186.25 & 6.00 & 185.34 & 6.00 \\
\hline & & & & & & & & 193.30 & 7.00 & 189.22 & 7.00 & 187.46 & 7.00 \\
\hline & & & & & & & & 194.87 & 8.00 & 191.00 & 8.00 & 189.58 & 8.00 \\
\hline & & & & & & & & 196.11 & 9.00 & 192.83 & 9.00 & 193.31 & 9.00 \\
\hline & & & & & & & & 196.89 & 10.00 & 197.11 & 10.00 & 195.54 & 10.00 \\
\hline & & & & & & & & 198.92 & 11.00 & 199.96 & 11.00 & 197.24 & 11.00 \\
\hline & & & & & & & & 201.55 & 12.00 & 203.95 & 12.00 & 200.94 & 12.00 \\
\hline & & & & & & & & 203.56 & 13.00 & 206.17 & 13.00 & 205.23 & 13.00 \\
\hline & & & & & & & & 205.57 & 14.00 & 208.39 & 14.00 & 207.23 & 14.00 \\
\hline & & & & & & & & 207.58 & 15.00 & 210.61 & 15.00 & 209.23 & 15.00 \\
\hline & & & & & & & & 209.59 & 16.00 & 211.07 & 16.00 & 211.23 & 16.00 \\
\hline & & & & & & & & 215.29 & 17.00 & 214.37 & 17.00 & 214.15 & 17.00 \\
\hline & & & & & & & & 222.25 & 18.00 & 218.07 & 17.79 & 219.85 & 17.79 \\
\hline & & & $\begin{array}{l}\text { : Left } \\
\text { Bank }\end{array}$ & & & & & $\begin{array}{l}\text { : Right } \\
\text { Bank }\end{array}$ & & & & & \\
\hline
\end{tabular}


TABLE 2.

COORdinAtes of River BEd AT CROSS SECTION No. KB63, DOWNSTREAM OF MENTURUS RubBer DAM

\begin{tabular}{|c|c|c|c|c|c|c|c|c|c|c|c|c|c|}
\hline \multicolumn{2}{|c|}{$\begin{array}{c}\text { Measurement } \\
\text { '92 } \\
\end{array}$} & \multicolumn{2}{|c|}{ Prediction '97 } & \multicolumn{2}{|c|}{ Prediction '01 } & \multicolumn{2}{|c|}{ Prediction '06 } & \multicolumn{2}{|c|}{ Prediction '08 } & \multicolumn{2}{|c|}{ Prediction '11 } & \multicolumn{2}{|c|}{ Measurement '11 } \\
\hline 0.00 & 18.00 & 0.00 & 18.00 & 0.00 & 18.00 & 0.00 & 18.00 & 0.00 & 18.00 & 0.00 & 18.00 & 0.00 & 18.00 \\
\hline 6.10 & 17.00 & 6.10 & 17.00 & 6.11 & 17.00 & 5.50 & 17.00 & 6.15 & 17.00 & 3.16 & 17.42 & 7.40 & 17.00 \\
\hline 10.89 & 16.00 & 10.89 & 16.00 & 12.27 & 16.00 & 21.75 & 16.00 & 7.96 & 16.00 & 4.25 & 17.00 & 18.87 & 16.10 \\
\hline 35.96 & 15.00 & 35.96 & 15.00 & 34.64 & 15.00 & 30.61 & 15.00 & 25.18 & 15.00 & 26.01 & 16.00 & 27.41 & 16.00 \\
\hline 41.57 & 14.00 & 41.57 & 14.00 & 37.25 & 14.00 & 31.20 & 14.00 & 26.71 & 14.00 & 34.53 & 15.00 & 32.02 & 15.81 \\
\hline 48.21 & 13.00 & 48.21 & 13.00 & 48.38 & 13.00 & 40.79 & 13.88 & 41.81 & 13.00 & 39.24 & 14.00 & 36.52 & 15.00 \\
\hline 54.72 & 12.00 & 54.72 & 12.00 & 56.67 & 12.00 & 45.78 & 12.90 & 50.65 & 12.00 & 48.55 & 13.00 & 42.16 & 14.00 \\
\hline 58.49 & 11.00 & 58.49 & 11.00 & 57.15 & 11.00 & 57.95 & 11.90 & 52.83 & 11.00 & 51.06 & 12.78 & 47.80 & 13.00 \\
\hline 59.42 & 10.00 & 59.42 & 10.01 & 57.68 & 10.05 & 57.95 & 10.90 & 53.98 & 10.00 & 52.13 & 12.00 & 50.16 & 12.58 \\
\hline 63.03 & 9.00 & 63.60 & 8.77 & 61.12 & 8.99 & 59.61 & 9.90 & 55.13 & 9.00 & 53.50 & 11.00 & 51.10 & 12.00 \\
\hline 72.18 & 8.00 & 72.18 & 7.19 & 65.70 & 7.69 & 60.44 & 8.62 & 56.28 & 8.00 & 54.87 & 10.00 & 52.57 & 11.00 \\
\hline 72.30 & 7.97 & 72.30 & 7.16 & 75.24 & 6.51 & 63.21 & 7.29 & 57.59 & 6.98 & 55.77 & 9.00 & 54.04 & 10.00 \\
\hline 72.80 & 8.00 & 72.80 & 7.19 & 76.54 & 6.43 & 68.46 & 5.02 & 59.05 & 5.97 & 57.41 & 8.00 & 55.51 & 9.00 \\
\hline 79.13 & 8.34 & 78.60 & 6.99 & 80.79 & 6.51 & 82.55 & 3.25 & 60.52 & 5.85 & 59.34 & 6.85 & 57.44 & 8.00 \\
\hline 94.39 & 8.00 & 98.06 & 6.65 & 84.51 & 6.59 & 104.51 & 4.28 & 63.87 & 5.13 & 61.77 & 6.43 & 60.06 & 7.00 \\
\hline 114.35 & 8.37 & 118.72 & 7.02 & 91.99 & 6.51 & 111.60 & 4.14 & 68.88 & 4.58 & 63.47 & 5.28 & 62.17 & 6.00 \\
\hline 122.03 & 8.62 & 121.84 & 7.20 & 102.47 & 6.46 & 119.42 & 3.66 & 70.59 & 4.03 & 64.07 & 5.34 & 65.25 & 5.00 \\
\hline 151.97 & 9.40 & 140.00 & 7.67 & 112.69 & 6.58 & 141.49 & 2.33 & 75.05 & 3.03 & 71.55 & 3.39 & 68.15 & 4.26 \\
\hline 158.45 & 9.26 & 151.34 & 8.75 & 121.90 & 6.43 & 148.13 & 3.74 & 76.59 & 2.72 & 81.99 & 2.73 & 70.43 & 3.52 \\
\hline 158.98 & 10.00 & 158.98 & 9.96 & 123.27 & 6.58 & 150.30 & 5.12 & 81.54 & 3.03 & 101.49 & 4.45 & 82.86 & 2.80 \\
\hline 159.94 & 11.00 & 159.94 & 11.00 & 138.43 & 6.75 & 153.22 & 7.90 & 94.89 & 3.29 & 113.31 & 4.07 & 95.81 & 3.52 \\
\hline 160.90 & 12.00 & 160.90 & 12.00 & 153.70 & 8.27 & 156.77 & 8.56 & 99.26 & 4.03 & 119.64 & 3.39 & 100.52 & 4.22 \\
\hline 162.03 & 13.00 & 162.03 & 13.00 & 155.31 & 8.83 & 157.11 & 8.84 & 105.50 & 4.66 & 124.62 & 3.32 & 122.40 & 3.52 \\
\hline 165.14 & 14.00 & 165.14 & 14.00 & 159.09 & 8.96 & 159.24 & 9.05 & 106.32 & 5.04 & 140.09 & 2.90 & 136.99 & 2.80 \\
\hline 166.78 & 15.00 & 166.78 & 15.00 & 161.46 & 9.98 & 160.52 & 10.00 & 108.26 & 4.66 & 141.68 & 2.82 & 141.83 & 3.02 \\
\hline 168.42 & 16.00 & 168.42 & 16.00 & 162.11 & 11.00 & 162.71 & 11.00 & 117.83 & 4.03 & 142.12 & 2.90 & 144.91 & 4.02 \\
\hline 170.06 & 17.00 & 170.06 & 17.00 & 162.78 & 12.00 & 164.17 & 12.00 & 127.77 & 3.03 & 146.79 & 4.45 & 146.91 & 4.30 \\
\hline \multirow[t]{18}{*}{180.34} & 18.00 & 180.34 & 18.00 & 163.71 & 13.00 & 165.15 & 13.00 & 136.19 & 2.37 & 148.25 & 5.38 & 147.98 & 5.02 \\
\hline & & & & 167.11 & 14.00 & 167.83 & 14.00 & 145.48 & 3.03 & 149.51 & 6.51 & 149.48 & 6.02 \\
\hline & & & & 168.81 & 15.00 & 169.28 & 15.00 & 147.19 & 4.03 & 151.32 & 7.09 & 150.98 & 7.02 \\
\hline & & & & 170.51 & 16.00 & 170.73 & 16.00 & 149.32 & 4.49 & 152.84 & 8.00 & 152.48 & 8.02 \\
\hline & & & & 172.21 & 17.00 & 172.18 & 17.00 & 150.28 & 5.97 & 154.73 & 8.40 & 155.12 & 8.86 \\
\hline & & & & 180.73 & 18.00 & 179.97 & 17.55 & 151.94 & 6.98 & 159.73 & 9.00 & 156.25 & 9.00 \\
\hline & & & & & & 180.72 & 18.00 & 154.40 & 8.00 & 162.84 & 10.00 & 159.62 & 9.37 \\
\hline & & & & & & & & 158.90 & 9.00 & 163.54 & 11.00 & 161.91 & 10.00 \\
\hline & & & & & & & & 162.59 & 10.00 & 166.60 & 11.81 & 162.47 & 10.15 \\
\hline & & & & & & & & 163.12 & 11.00 & 167.86 & 13.00 & 163.37 & 11.00 \\
\hline & & & & & & & & 163.65 & 12.00 & 167.86 & 14.00 & 164.76 & 12.00 \\
\hline & & & & & & & & 165.88 & 13.00 & 168.75 & 15.00 & 166.73 & 13.00 \\
\hline & & & & & & & & 166.92 & 14.00 & 170.21 & 16.00 & 167.88 & 14.00 \\
\hline & & & & & & & & 168.35 & 15.00 & 171.67 & 17.00 & 169.15 & 15.00 \\
\hline & & & & & & & & 169.95 & 16.00 & 172.31 & 17.44 & 170.63 & 16.00 \\
\hline & & & & & & & & 171.69 & 17.00 & 180.06 & 17.80 & 172.11 & 17.00 \\
\hline & & & & & & & & 180.62 & 18.00 & 180.55 & 18.00 & 180.60 & 18.00 \\
\hline & & \multicolumn{4}{|c|}{ : Left Bank } & & & \multicolumn{2}{|c|}{ : Right Bank } & & & & \\
\hline
\end{tabular}

